\title{
An Experimental Investigation: Effect of Phase Inversion Methods on Membrane Structure and Its Performance on PEG Filtration
}

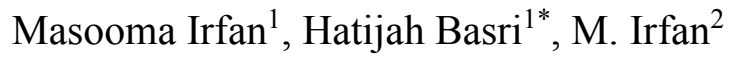 \\ ${ }^{1}$ Department of Technology and Heritage, Faculty of Science, Technology and \\ Human Development, Universiti Tun Hussein Onn Malaysia, Parit Raja, Batu Pahat \\ 86400, Johor, Malaysia \\ ${ }^{2}$ Department of Bioprocess Engineering, Faculty of Chemical Engineering, c/o \\ Institute of Bioproduct Development, Universiti Teknologi Malaysia, 81310 UTM \\ Johor Bahru, Johor, Malaysia
}

\begin{abstract}
In this work, the effect of different phase inversion process on membrane morphology and performance was studied. Polyethersulfone (PES) based polymeric membranes was fabricated containing polyvinylpyrrolidone (PVP) and carboxylic functionalized multiwall carbon nanotubes (MWCNT) as additives and polyethylene glycol (PEG) having a molecular weight $1 \mathrm{~K}, 10 \mathrm{~K}$ and $35 \mathrm{~K}$ (Dalton) were used as a model solution for observing the rejection/filteration ability of fabricated membranes. Non-solvent induce phase separation (NIP) and dry-wet phase separation (DWP) method was adopted for membrane synthesis. The FTIR spectra showed that PVP/MWCNT was effectively blended with PES polymer and different phase inversion method led to different internal morphologies of membranes as confirmed by FESEM images. The PEG rejection results suggested that membranes formed by DWP method had approximately double rejection ability than membranes formed by NIP process.
\end{abstract}

Keywords: Phase inversion, PES, asymmetric, rejection

\subsection{INTRODUCTION}

Development in the membrane separation techniques has become an important aspect due to its removal efficiency and low operating cost. Membrane is fabricated by different phase inversion processes are mostly used for waste water treatment and ultrafiltration application. A range of polymers such as poly(ethersulfone) (PES) [1], poly(sulfone) (PSf) [2], Poly(vinylidenefluoride) [3], cellulose acetate [4], poly(acrylonitrile [5] or their blends [6] have been used to prepare membranes with required composition and properties.

Phase inversion can be explained as a demixing procedure in which the homogeneous polymer solution is altered from a liquid to a solid state in a controlled way [7]. This transformation can be described in a number of ways [8], as Immersion precipitation, thermally induced phase separation, evaporation-induced phase separation, vapor-induced phase separation $[9,10]$. Research on symmetric or asymmetric membranes by solvent evaporation or by the wet phase inversion and dry-wet phase inversion (DWP) method have been extensively reported for flat sheet or hollow-fibre preparation. Among all mentioned methods Non-solvent induced phase separation (NIP) is dominating for fabrication of polymeric UF membrane mainly in PSf and PES at industrial level [11]. PVP have been broadly used as additives in fabrication of PES ultrafiltration (UF) membranes via phase separation methods. A smaller amount of macrovoid structure

* Corresponding to: H. Basri (email: hatijah@uthm.edu.my) 
and delayed demixing is due to the combination of PVP-PES with solvent into casting solution. It was reported, a large number of macrovoids formed by adding the dimethylformamide (DMF) into PVP/PES solution accompanied by increase water permeability and molecular weight cut off (MWCO) in membrane [12-14].

PVP increased the hydrophilicity of PES and enhanced the membrane actual performance due to its highly polar, non-ionic, inert and amphiphilic nature [15-17]. PVP was also reported as surfactant to decrease the coagulation effect and increase the dispersion capacity of MWCNT in different solvents [18]. Although, MWCNTs are hydrophobic but acid functionalization of MWCNT assist to create - $\mathrm{COOH}$ and $-\mathrm{OH}$ founctional groups on its surface that improve the bonding behaviour towards hydrophilic molecules as well $[19,20]$.

In this article, carboxylic and hydroxyl functional groups containing multiwall carbon nanotubes (cMWCNT) was blended togather with PVP and nanocomposites (NCs) were formed as per method mention in our previous study [21], then the NCs are blended with PES polymer to make different dope solutions (Table 1). The focus of this work was to investigate the effect of NIP and DWP processes on membrane structure and filtration of different molecular weight $(1 \mathrm{~K}, 10 \mathrm{~K}$ and $35 \mathrm{~K}$ ) polyethylene glycol solutions. The resultant membranes were characterized by FTIR, and FESEM, whereas filtration performances of fabricated membranes were evaluated by different PEG aqueous solution via cross flow cells.
Table 1 Formulation of membranes

\begin{tabular}{cllll}
\hline *Membrane & $\begin{array}{l}\text { f- } \\
\text { MWCNT }\end{array}$ & PVP & PES & DMF \\
\hline M-NIP & 0.3 & 3 & 16 & 80.70 \\
M-DWP & 0.3 & 3 & 16 & 80.70 \\
\hline *M-NIP stands for membrane formed by non-solvent \\
induce phase inversion process, whereas M-DWP for \\
membranes fabricated by dry-wet phase inversion.
\end{tabular}

\subsection{EXPERIMENTAL}

\subsection{Materials}

PES, Ultrason E6020P) having a weight-average molecular weight 58,000 was purchased from BASF, Germany. DMF [HCON (CH3) 2; weight-average molecular weight $=$ $80.14 \mathrm{~g} / \mathrm{mole}]$ of $99.8 \%$ purity was purchased from Labscan Asia Co., Ltd. Reagent grade PVP-K90, Polyethylene glycol (PEG) $(\mathrm{MW}=1 \mathrm{~K}, 10 \mathrm{~K}$ and $35 \mathrm{~K}$ Dalton) was obtained from Fluka; and Acid functionalized MWCNTs (color: black, purity: $>98 \%$, length: 15 micrometer, average diameter: $11 \mathrm{~nm}$ and true density: $0.04 \mathrm{~g} / \mathrm{cm} 3)$ was purchased from Chengdong (China).

\subsection{Membrane Fabrication}

The conventional heating method was applied to make different dope solutions as per the formulation shown in Table 1. The PES and (fMWCNT/PVP) based dope solution was dispensed into the storage bottle and ultrasonic bath was used to remove the air bubbles. In this study, nonsolvent induced phase separation (NIP) and dry-wet phase separation (DWP) methods were used in the production of polymeric membranes. The flat sheet membranes were cast by casting knife having thickness $180-200 \mu \mathrm{m}$ onto a glass plate at room temperature. In the NIP method, immediately after that casting the membranes, the glass plates were immersed in a deionized water 
coagulation bath at room temperature. After $5 \mathrm{~min}$, the fabricated films were put in the second bath with fresh distilled water for $24 \mathrm{~h}$ to ensure that the solvent is leached and phase inversion is completely done. In DWP process, after casting the membrane, they were coagulated by exposure to air for a certain time and then dipped in a coagulation bath, containing water as a nonsolvent for at least $12 \mathrm{~h}$. Finally, the prepared membranes were dried by placing them between two filter papers.

\subsection{Characterization}

The FTIR (Perkin-Elmer Spectrum One B) instrument was used for the verification of the presence of PES and $\mathrm{PVP} / \mathrm{f}-\mathrm{MWCNT}$ in the membrane matrix. FTIR measurements were carried out in purified $\mathrm{KBr}$ (water less) pellets and the scanning range of the experiment was $375-4000 \mathrm{~cm}^{-1}$. The morphology of a cross section of membranes was observed using FESEM (JEOL JSM-7500F). The membrane sample was snapped in liquid nitrogen and then sputter-coated with platinum and mounted onto brass plates using double-sided cellophane tapes in a lateral position.

\subsection{PEG Filtration}

The separation performances of fabricated flat sheet membranes were evaluated by studying the rejection rate of different molecular weights (1000, 10,000 and 35,000 Dalton) PEG solutions having concentration $1000 \mathrm{ppm}$ in distilled water at 5 bar pressure. The cross flow cell having an effective area of $42 \mathrm{~cm}^{2}$ was used, whereas the rejection rate was calculated by Eq. 1,

$$
\mathrm{R}=\left(1-\frac{\mathrm{C}_{\mathrm{p}}}{\mathrm{C}_{\mathrm{f}}}\right) \times 100 \%
$$

where $\mathrm{Cp}$ and $\mathrm{Cf}$ are the concentrations of the permeate and feed respectively.
Modified Dragendorff reagent method was used to detect PEG concentrations in solution [22].

\subsection{RESULTS AND DISCUSSION}

The FTIR spectra of fabricated membranes are illustrated by Figure 1 . The peaks at 3082 and $3544 \mathrm{~cm}^{-1}$ belonged to the alcohol and $-\mathrm{OH}$ group of carboxylic of MWCNT, whereas the bands at $1663.49 \mathrm{~cm}^{-1}$ represented the tertiary amide of PVP [23]. The existence of PES polymer were verified by the sharp peaks at 1244 and 1149 $\mathrm{cm}^{-1}$, were representing the $\mathrm{S}=\mathrm{O}$ asymmetric and symmetric vibrations respectively and peaks at 1481 and $1576 \mathrm{~cm}^{-1}$ corresponded to the bending vibration of PES aromatic rings [24]. Thus, the presence of "PES and PVP/fMWCNT" are confirmed in the membrane formulations by FTIR.

Figure 2 represents the FESEM images of the membranes, formed by NIP (M-NIP) and DWP (M-DWP) process. Although the chemical formulation of both the membranes were same but different morphologies were observed, showed that selection of proper phase inversion method is very important to develop the desired internal structure in membranes. In the "M-NIP" characteristic asymmetric morphological structure consists of a finger like-entities was observed [25, 26], whereas M-DWP showed the sponge-like structure that was more close the symmetric morphological classification of membranes.

The presence of the non-solvent (water and air) in the dope solution during phase inversion, not only changes thermodynamic state of dope solution, but also differently influenced the conformation and dynamics of the polymer, which in turn affects the kinetics of phase separation. Generally, delay phase inversion led to sponge-like 
structures in membrane formation. In the NIP, the exchange rate of solvent

cross flow cell through fabricated
membranes.

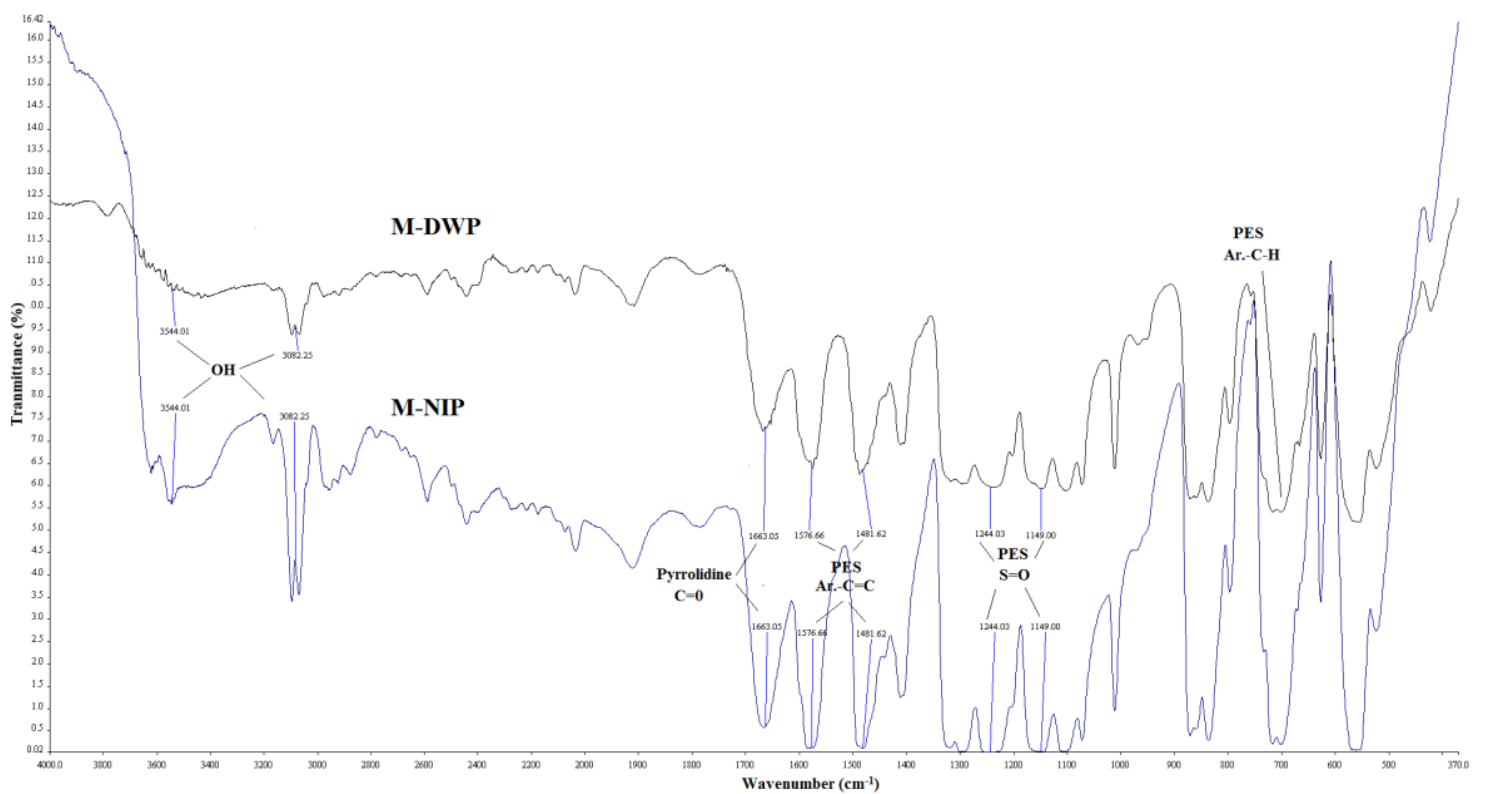

Figure 1 The FTIR spectra of M-NIP and M-DWP membranes

and dope solution with non-solvent (water) was very fast as compared to the DWP process which took nearly $5 \mathrm{~min}$ for exchange of solvent with air, thus the shorter time of phase inversion lead to asymmetric structure as in M-NIP and higher time to spongy morphologies (M-DWP).

Figure 3 represents the rejectionfiltration of $1 \mathrm{~K}, 10 \mathrm{~K}$ and $35 \mathrm{~K}$ molecular weight PEG solutions via performances of membranes for different molecular weight PEG depend on the corresponding pore size and its internal structures. The asymmetric membranes generally have larger pore size and their finger like capillaries, help and lead to faster filteration for the solutes as compared to spongy structured membranes. In spongy membranes, the alignment of pores and the absence of large capillaries resists

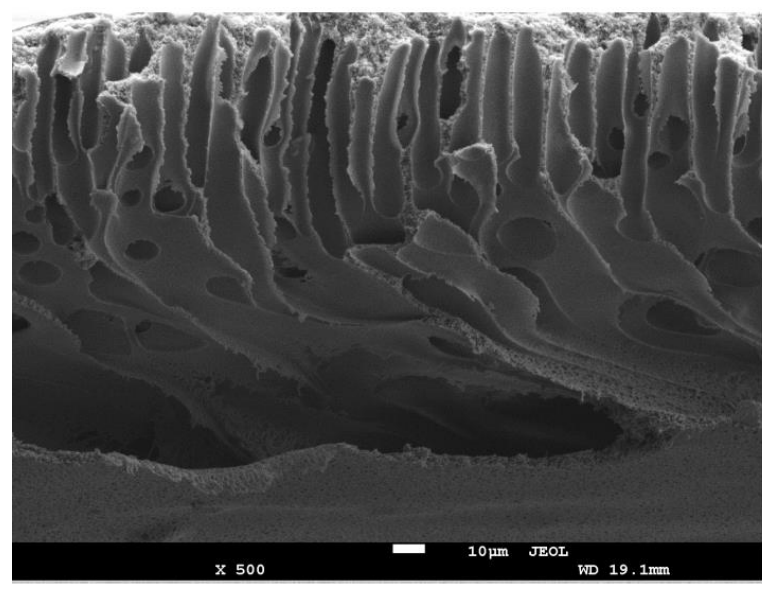

M-NIP

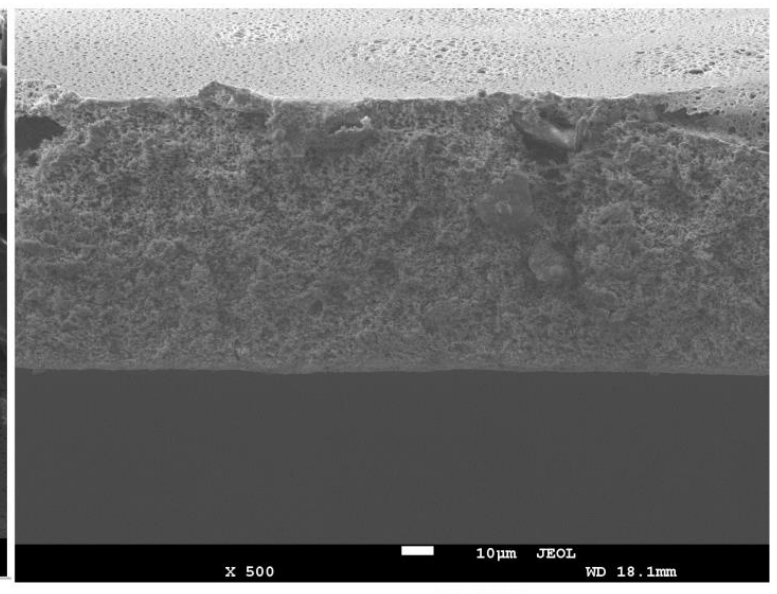

M-DWP

Figure 2 The FESEM images of formulated membranes. NIP and DWP stand for non-solvent induce phase separation and dry-wet induce phase separation respectively 


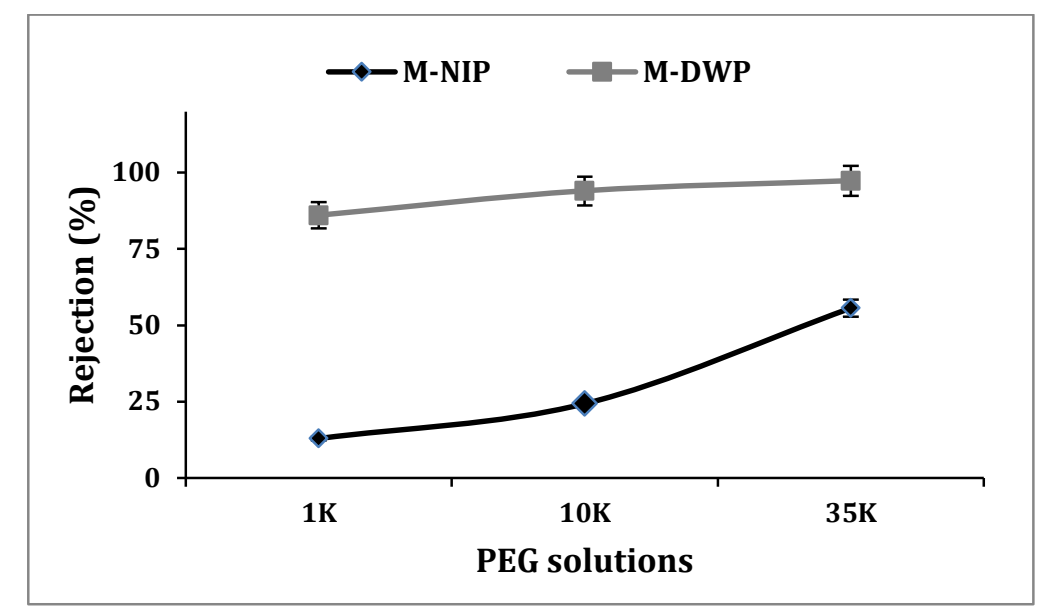

Figure 3 The rejection ratio of different PEG solutions through fabricated membranes

the PEG filtration in each place across the membranes. In the Figure 3, the MNIP shows lower clearance ratio for all PEG solutions (Maximum 55.6\% with PEG 35K) as compared to M-DWP membranes, where the M-DWP membranes shows more than 90\% rejection rate with $\mathrm{PEG} 10 \mathrm{~K}$ and $35 \mathrm{~K}$ solutions. The high rejection ratio of PEG in M-DWP membranes were obtained due to its spongy structures that highly resisted the flow of PEG solutes through the membrane.

\subsection{CONCLUSION}

The carboxylic functionalized MWCNT and PVP based additives were successfully incorporated with PES polymers in both the membranes as confirmed by FTIR. The adoption of different phase inversion methods led to different morphologies of fabricated membranes that showed different results for the PEG rejection. These results suggested that the selection of the phase inversion process plays a very important role in the development of internal morphology of membranes which in turn affect the filtration performances of specific solute.

\section{REFERENCES}

[1] Amirilargani, M., M. Sadrzadeh, and T. Mohammadi. 2010. Synthesis and characterization of polyethersulfone membranes. Journal of Polymer Research. 17(3): 363-377.

[2] Chakrabarty, B., A. Ghoshal, and M. Purkait. 2008. Preparation, characterization and performance studies of polysulfone membranes using PVP as an additive. Journal of Membrane Science. 315(1): 36-47.

[3] Zuo, D.-y., et al. 2008. The influence of PEG molecular weight on morphologies and properties of PVDF asymmetric membranes. Chinese Journal of Polymer Science. 26(04): 405414.

[4] Saljoughi, E. and T. Mohammadi. 2009. Cellulose acetate (CA)/polyvinylpyrrolidone (PVP) blend asymmetric membranes: Preparation, morphology and performance. Desalination. 249(2): 850-854.

[5] Yang, S. and Z. Liu. 2003. Preparation and characterization of polyacrylonitrile ultrafiltration membranes. Journal of Membrane Science. 222(1): 8798. 
[6] Amirilargani, M., A. Sabetghadam, and T. Mohammadi. 2012. Polyethersulfone/polyacrylonitril e blend ultrafiltration membranes with different molecular weight of polyethylene glycol: preparation, morphology and antifouling properties. Polymers for Advanced Technologies. 23(3): 398-407.

[7] Drioli, E. and L. Giorno. 2009. Membrane Operations: Innovative Separations and Transformations. Wiley-VCH

[8] Baker, R. W. 2000. Membrane Technology. Wiley Online Library.

[9] Liu, F., et al. 2011. Progress in the production and modification of PVDF membranes. Journal of Membrane Science. 375(1): 1-27.

[10] Pinnau, I. and B. Freeman. 2000. Formation and modification of polymeric membranes: overview. Membrane Formation and Modification . 744: 1-22.

[11] Lalia, B. S., et al. 2013. A review on membrane fabrication: Structure, properties and performance relationship. Desalination. 326: 77-95.

[12] Boom, R., et al. 1992. Microstructures in phase inversion membranes. Part 2. The role of a polymeric additive. Journal of Membrane Science. 73(2): 277-292.

[13] Mosqueda-Jimenez, D., et al. 2004. Influence of processing conditions on the properties of ultrafiltration membranes. Journal of Membrane Science. 231(1): 209-224

[14] Yeo, H.-T., S.-T. Lee, and M.-J. Han. 2000. Role of a Polymer Additive in Casting Solution in Preparation of Phase Inversion Polysulfone Membranes. Journal of Chemical Engineering of Japan. 33(1): 180-184

[15] Yang, Q., T.-S. Chung, and M. Weber. 2009. Microscopic behavior of polyvinylpyrrolidonehydrophilizi ng agents on phase inversion polyethersulfone hollow fiber membranes for hemofiltration. Journal of Membrane Science. 326(2): 322-331

[16] Sun, M., et al. 2009. Improved antifouling property of PES ultrafiltration membranes using additive of silica- PVP nanocomposite. Industrial \& Engineering Chemistry Research. 49(2): 790-796.

[17] Barzin, J., et al. 2004. Effect of polyvinylpyrrolidone on morphology and performance of hemodialysis membranes prepared from polyether sulfone. Journal of Applied Polymer Science. 92(6): 3804-3813.

[18] Kim, S. W., et al. 2012. Surface modifications for the effective dispersion of carbon nanotubes in solvents and polymers. Carbon. 50(1): 3-33

[19] Niyogi, S., et al. 2002. Chemistry of single-walled carbon nanotubes. Accounts of Chemical Research. 35(12): 1105-1113

[20] Raravikar, N. R., et al. 2005. Synthesis and characterization of thickness-aligned carbon nanotube-polymer composite films. Chemistry of Materials. 17(5): 974-983.

[21] Irfan, M., et al. 2014. Surface modification and performance enhancement of nano-hybrid $\mathrm{f}$ MWCNT/PVP90/PES

hemodialysis membranes. Journal of Membrane Science. 467: 73-84.

[22] Jia, Z. and C. Tian. 2009. Quantitative determination of polyethylene glycol with 
modified Dragendorff reagent method. Desalination. 247(1): 423-429.

[23] Caminati, W., et al. 1998. Free Jet Absorption Millimeter Wave Spectrum of Pyrrolidine: Assignment of a Second, Equatorial, the Most Stable Conformer. Journal of Molecular Spectroscopy. 191(1): 45-48

[24] Choi, J.-H., J. Jegal, and W.-N. Kim. 2006. Fabrication and characterization of multi-walled carbon nanotubes/polymer blend membranes. Journal of Membrane Science. 284(1): 406415
[25] Taniguchi, M., J. E. Kilduff, and G. Belfort. 2003. Low fouling synthetic membranes by UVassisted graft polymerization: monomer selection to mitigate fouling by natural organic matter. Journal of Membrane Science. 222(1): 59-70.

[26] Taniguchi, M. and G. Belfort. 2004. Low protein fouling synthetic membranes by UVassisted surface grafting modification: varying monomer type. Journal of Membrane Science. 231(1): 147-157. 